Artículo Original

\title{
Factores socio-laborales asociados a padecer ansiedad, depresión y estrés en profesionales de la salud de la serranía peruana durante la pandemia de la COVID-19
}

\section{Socio-occupational factors associated with anxiety, depression and stress in health professionals of the Peruvian highlands during the COVID-19 pandemic}

https://doi.org/10.52808/bmsa.7e5.61e2.011

Fleming Ambrosio ${ }^{1 *}$

https://orcid.org/0000-0002-0132-4870

Grace Torres-Santos 1

https://orcid.org/0000-0002-9854-6911

Lipselotte de Jesús Infante Rivera ${ }^{1}$

https://orcid.org/0000-0001-6094-1070

J. Franco Rodríguez-Alarcon ${ }^{2,3}$

https://orcid.org/0000-0003-4059-8214

Christian R. Mejia ${ }^{4}$

Recibido: 23/03/2021

https://orcid.org/0000-0002-5940-7281

Aceptado: 17/06/2021

\section{RESUMEN}

La COVID-19 ha generado una pandemia sin precedentes. Este escenario podría afectar la salud mental del personal sanitario, influyendo en su desempeño laboral con la posibilidad de dejar secuelas a largo plazo. El objetivo fue determinar los factores socio-laborales asociados a padecer ansiedad, depresión y estrés en profesionales de la salud de la serranía peruana durante la pandemia. Estudio transversal analítico. Se encuestó de forma virtual a médicos y otros profesionales del Hospital Nacional Ramiro Prialé Prialé, en la sierra peruana. La depresión, ansiedad y estrés se midieron con la escala DASS-21; a estos se les asoció distintas variables socio-laborales. Tuvieron más depresión severa a mayor edad y si algún familiar se había enfermado en casa, pero menos depresión severa los que tuvieron hijos, los que tuvieron más años de ejercicio profesional y al tener seguro social. Tuvieron menos ansiedad los que tuvieron hijos; menos ansiedad severa y los que tuvieron a un familiar fuera de casa que se enfermó; por el contrario, tuvieron mayor ansiedad moderada los que trabajaban más horas al día, severa si es que el encuestado se había enfermado y de ambos tipos si es que algún familiar había fallecido. Tuvieron más estrés los que trabajaban más horas al día y los que tenían un familiar fallecido. Las características más relevantes del personal de salud con problemas de su salud mental fueron tener mayor edad, antecedente familiar de COVID-19, antecedente de muerte de un familiar por COVID-19 y el tener más horas de trabajo.

Palabras clave: coronavirus, equipo de protección personal, salud mental, personal de salud, salud ocupacional, Latinoamérica.

\begin{abstract}
COVID-19 has generated an unprecedented pandemic. This scenario could affect the mental health of healthcare personnel, influencing their work performance with the possibility of leaving long-term sequelae. The objective was to determine the socio-occupational factors associated with suffering from anxiety, depression and stress in health professionals in the Peruvian highlands during the pandemic. Cross-sectional study. Doctors and other professionals at the Ramiro Prialé Priale National Hospital in the Peruvian highlands were surveyed virtually. Depression, anxiety and stress were measured with the DASS-21 scale; these were associated with different socio-labour variables. More severe depression was found at older ages and if a family member had been ill at home, but less severe depression was found among those who had children, those who had more years of professional practice and those who had social security. Those who had children had less anxiety; less severe anxiety and those who had a relative away from home who became ill; on the other hand, those who worked more hours per day had more moderate anxiety, severe anxiety if the respondent had become ill and both types if a family member had died had more moderate anxiety. Those who worked more hours per day and those who had a deceased family member had more stress. The most relevant characteristics of health workers with mental health problems were older age, family history of COVID-19, history of death of a family member from COVID-19 and longer working hours.
\end{abstract}

Key words: coronavirus, personal protection equipment, mental health, health personnel, occupational health, Latin America.

1. Universidad Continental. Huancayo, Perú 2. Asociación Médica de Investigación y Servicios en Salud. Lima, Perú.

3. Universidad Ricardo Palma. Lima, Perú. 4. Centro de Investigación en Medicina Traslacional. Universidad Norbert Wiener. Lima, Perú.

\section{Introducción}

*Autor de Correspondencia: fleambhor@gmail.com

Los coronavirus son importantes patógenos que han afectado principalmente al sistema respiratorio de los seres humanos (Chang et al., 2016). A finales del 2019 se identificó una nueva cepa como la causa de un brote de casos 
de neumonía viral en Wuhan, una ciudad en la provincia de Hubei-China (Li et al., 2020 a; Organización Panamericana de la Salud, 2020). Esta infección se extendió rápidamente, generando una epidemia en dicho país, seguida de un aumento exponencial de casos a nivel mundial (Organización Mundial de la Salud, 2020). A fines del mes de enero, la Organización Mundial de la Salud (OMS) designó la enfermedad como "COVID-19" (Coronavirus Disease 2019), y posteriormente se le consideró como pandemia (Epidemiology Working Group for NCIP Epidemic Response \& Chinese Center for Disease Control and Prevention, 2020; Zhu et al., 2020).

En el contexto de esta pandemia, el personal de salud o sanitario (PS) se ha visto envuelto en nuevos retos, como mayores horas de jornada laboral (Ministerio de Salud, 2020), alto riesgo de contagio por mayor exposición (Koh, 2020), la posibilidad de ser portadores asintomáticos -con el respectivo riesgo de infección en sus hogares- (Chen et al., 2020; Lagasse, 2020), la escasez de equipos de protección personal (EPP) (Greenberg et al., 2020), el sentimiento del apoyo inadecuado (Rolim Neto et al., 2020), las lesiones morales (Greenberg et al., 2020), la pena y culpa por las muertes (Rolim Neto et al., 2020); entre otros muchos factores que estarían afectando su salud mental. Todo en conjunto provocaría altas cargas de estrés, ansiedad, depresión y desmotivación (Kang et al., 2020 a; Lai et al., 2020). Habiendo sido reportado esto sobre todo entre los que estuvieron en la primera línea (Kang et al., 2020 b); con efectos directos e indirectos ( $\mathrm{Li}$ et al., 2020 b). Incluso, con la presentación de trastornos de estrés postraumáticos (TEPT) (Torales et al., 2020).

En general, todo este panorama y la incertidumbre que se generó (Huang et al., 2020), sumado a todas las carencias (Rolim Neto et al., 2020; Santarone et al., 2020), han repercutido de diversas formas (Liang et al., 2020). Sin embargo, son aún pocos los estudios latinoamericanos que abordan el tema (Lozano-Vargas, 2020; Ornell et al., 2020). Y la mayoría se han realizado en las principales ciudades de la costa, pero no se ha encontrado hasta el momento el reporte de una población que vive en la altura geográfica de los andes. Por tal motivo, el objetivo de nuestro estudio fue el determinar los factores socio-laborales asociados a padecer ansiedad, depresión y estrés en profesionales de la salud de la serranía peruana durante la pandemia.

\section{Materiales y Métodos}

La investigación realizó un estudio de tipo observacional, analítico, transversal y prospectivo. La población especifica total de esta institución está conformada por el personal sanitario (médicos, enfermeras, obstetras, técnicas en enfermería, nutricionistas, biólogos, tecnólogos médicos, químicos farmacéuticos, etc.) y que laboren tanto en áreas COVID-19 o no COVID-19. El estudio se realizó en marco de la pandemia por la COVID-19, durante el estado de emergencia de la primera ola. Se realizó el cálculo de tamaño muestral con un piloto previo, en el que se encontró una mínima diferencia de proporciones de 7,5\% (46,5\% versus 54,0\%), para una potencia del $80 \%$, nivel de confianza del 95\% y para una muestra única, con lo que se obtuvo que se requería 347 participantes. La muestra fue obtenida de manera no probabilística a través de encuestado virtual. Se captó a los participantes por muestreo de bola de nieve (para esto se contactó con algunos conocidos, luego estos nos refirieron a otros y así hasta llegar al tamaño mínimo muestral).

Se incluyó a los participantes que laboraran en el Hospital Nacional Ramiro Prialé Prialé, sin importar su condición laboral (nombrado o contratado), que sea personal de salud (entre los grupos mencionados previamente), que tenga por lo menos 3 meses laborando en el hospital durante la pandemia y que hayan deseado ser parte del estudio. Se excluyó a los estudiantes de medicina que estén realizando externado o internado, al personal de salud del hospital nombrado o contratado que esté cumpliendo labores administrativas, al personal de salud nombrado o contratado que no esté laborando en el hospital durante el desarrollo del estudio (descanso médicos o licencias) y aquellos que decidieron participar pero que no completaron la encuesta adecuadamente; se tuvo menos de 20 fichas excluidas.

El instrumento constó de 2 secciones: primero las características socio-demográficas, segundo la evaluación de la salud mental, donde se usó el cuestionario para medir depresión, ansiedad y estrés (Depression, anxiety and stress scales: DASS-21), que consta de 21 ítems (Daza et al., 2002). Se consideró a estas tres patologías como las variables "outcome", para el análisis se tomó en cuenta su presentación moderada o mayor (que incluía a las presentaciones: moderada + severa + muy severa) y severa o mayor (que incluía a las presentaciones: severa + muy severa). Se dividió a las variables socio-demográficas en dos categorías: socio-demográficas "per se" y la relación personal o familiar con el COVID-19. Las variables socio-demográficas fueron: sexo, edad, si tenía hijos, los años que ejercía su profesión, la cantidad de horas de trabajo diario, si contaba con un seguro médico social y la ocupación dentro del hospital. Las variables de relación con el COVID-19 fueron: antecedente personal de enfermarse, si una amistad cercana se enfermó, si un familiar que vive en casa sufrió la enfermedad, si un familiar que no vive en casa se enfermó y si algún familiar falleció a causa de la patología.

El equipo de investigación desarrolló el protocolo del estudio, este protocolo fue presentado al comité de ética del Hospital Nacional Ramiro Prialé Prialé (HNRPP). Después de su aprobación, se procedió al encuestado, esto a través de una hoja de formulario de Google Drive; esto mediante un instrumento auto aplicado (debido a la restricción 
para el encuestado presencial por la emergencia sanitaria). Durante el enrolamiento de los participantes se solicitó el consentimiento verbal de los participantes; esto como requisito para que formen parte del estudio.

El estudio se desarrolló durante las primeras semanas de la pandemia; es decir, cuando empezaron a llegar los primeros casos al hospital (durante la primera ola de la enfermedad: abril-junio). Al finalizar la recolección de datos se procedió a crear una base de datos, para lo cual se utilizó una hoja del programa Microsoft Excel 2019. Al finalizar, se procedió a realizar un control de calidad de los datos, esto mediante la verificación de dos de los autores. Con la información corroborada y revisada, se procedió a realizar los procedimientos de análisis estadístico.

Para procesar los datos se realizó un análisis descriptivo de la base y se obtuvo las frecuencias y porcentajes de las variables categóricas, así como, las medidas de tendencia central y de dispersión (según la evaluación de la normalidad de las variables cuantitativas). Para la estadística analítica se realizó un análisis bivariado y multivariado, para esto se usó los modelos lineales generalizados, con la familia Poisson, la función de enlace log, ajustes para varianzas robustas y según la ocupación. Con todo esto se obtuvo las razones de prevalencia; los intervalos de confianza al 95\% y los valores p. Para todos los valores estadísticos se consideró el 0,05 como punto de corte para determinar la significancia estadística.

El presente proyecto pasó por revisión y aprobación del comité de ética del HNRPP. En todo momento se guardó la confidencialidad del participante, no fue divulgada, se anonimizó las respuestas, esto mediante la colocación de un código (para que la base con la que se trabajó no tenga los datos identificatorios). También se le explicó a los encuestados que los resultados de la encuesta no tendrían repercusión alguna en su ámbito laboral; esto según a lo coordinado con el comité de ética.

\section{Resultados}

De los 353 encuestados, el 53,0\% (187) eran mujeres, la mediana de edad fue 41 años (rango intercuartílico: 34-50 años), el 59,1\% (208) eran médicos, tenían 8 años de mediana de ejercicio profesional (rango intercuartílico: 3-14 años), el 85,0\% (300) aún no se había enfermado por COVID-19 (Tabla 1).

Tabla 1. Características de los profesionales de la salud en Huancayo-Perú

\begin{tabular}{|c|c|c|}
\hline Variable & Frecuencia & Porcentaje \\
\hline \multicolumn{3}{|l|}{ Sexo } \\
\hline Femenino & 187 & $53,0 \%$ \\
\hline Masculino & 166 & $47,0 \%$ \\
\hline \multicolumn{3}{|l|}{ Edad (años) } \\
\hline Media y desviación estándar & 42,5 & 11,4 \\
\hline Mediana y rangos intercuartílicos & 41 & $34-50$ \\
\hline \multicolumn{3}{|l|}{ Tiene hijos } \\
\hline No & 112 & $31,7 \%$ \\
\hline $\mathrm{Si}$ & 241 & $68,3 \%$ \\
\hline \multicolumn{3}{|l|}{ Ocupación } \\
\hline Médico & 208 & $59,1 \%$ \\
\hline Enfermera o técnica de enfermería & 92 & $26,1 \%$ \\
\hline Psicólogo & 12 & $3,4 \%$ \\
\hline Otro & 40 & $11,4 \%$ \\
\hline \multicolumn{3}{|l|}{ Años que ejerce su profesión } \\
\hline Media y desviación estándar & 10,1 & 9,5 \\
\hline Mediana y rangos intercuartílicos & 8 & $3-14$ \\
\hline \multicolumn{3}{|l|}{ Horas que trabaja al día } \\
\hline Media y desviación estándar & 8,4 & 2,8 \\
\hline Mediana y rangos intercuartílicos & 8 & $6-12$ \\
\hline \multicolumn{3}{|l|}{ Tiene seguro social } \\
\hline No & 13 & $3,7 \%$ \\
\hline $\mathrm{Si}$ & 340 & $96,3 \%$ \\
\hline \multicolumn{3}{|l|}{ Me enfermé yo de COVID-19 } \\
\hline No & 300 & $85,0 \%$ \\
\hline $\mathrm{Si}$ & 53 & $15,0 \%$ \\
\hline \multicolumn{3}{|l|}{ Un amigo se enfermó con COVID-19 } \\
\hline No & 146 & $41,4 \%$ \\
\hline $\mathrm{Si}$ & 207 & $58,6 \%$ \\
\hline \multicolumn{3}{|l|}{ Algún familiar en casa se enfermó } \\
\hline No & 267 & $75,6 \%$ \\
\hline $\mathrm{Si}$ & 86 & $24,4 \%$ \\
\hline \multicolumn{3}{|l|}{ Familiar fuera de casa se enfermó } \\
\hline No & 248 & $70,3 \%$ \\
\hline $\mathrm{Si}$ & 105 & $29,7 \%$ \\
\hline \multicolumn{3}{|l|}{ Algún familiar falleció por COVID-19 } \\
\hline No & 278 & $79,0 \%$ \\
\hline $\mathrm{Si}$ & 74 & $21,0 \%$ \\
\hline
\end{tabular}


La distribución de los participantes según los resultados de su evaluación psicológica nos mostró que la mayor parte de los participantes se encontraban en los rangos de normalidad con respecto a la depresión, ansiedad y estrés. En el caso de la ansiedad, se encontró que 19 participantes se encontraban en un grado extremadamente severo. Con respecto a la depresión, después de la normalidad (o no tener la enfermedad), el segundo mayor grupo se encontraba entre aquellos que presentaron niveles bajos de depresión (37 participantes). Y en el caso del estrés, los grupos más relevantes fueron los que tenían estrés bajo (27 participantes) y estrés moderado (26 participantes), sin contar a los que estuvieron sanos (Figura 1).

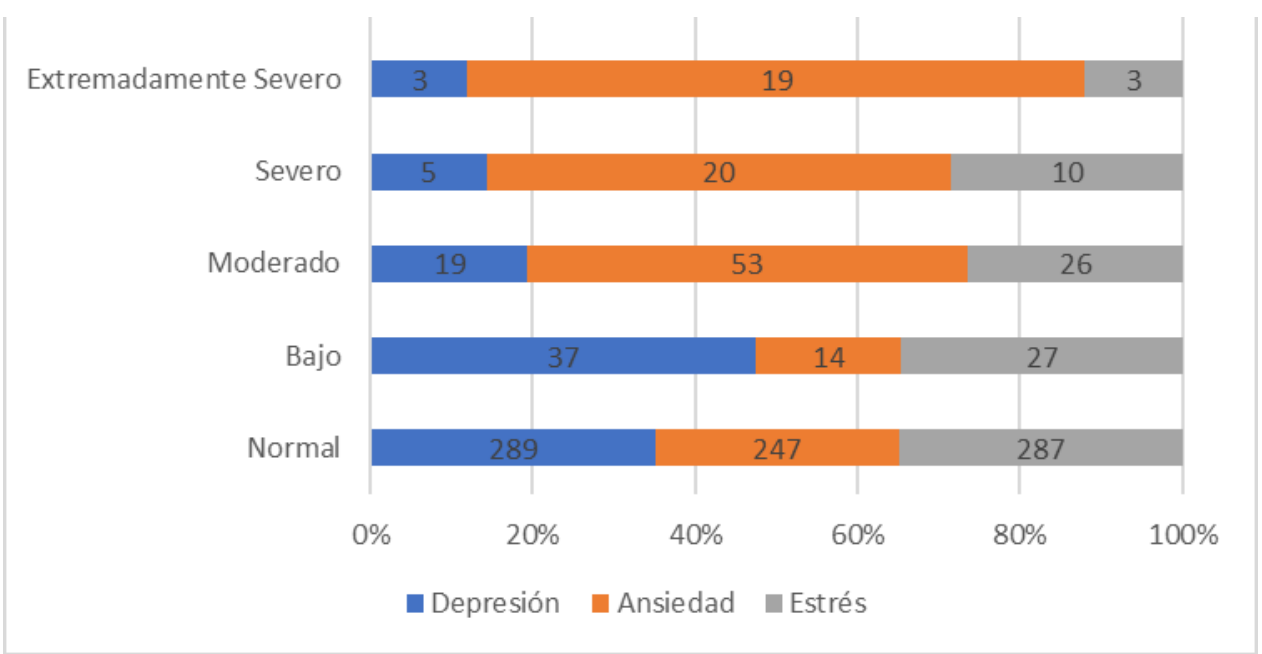

Figura 1. Distribución de los participantes según depresión, ansiedad y estrés

En el análisis multivariado, tuvieron más depresión severa a mayor edad (valor p=0,017) y si algún familiar se había enfermado en casa $(\mathrm{p}<0,001)$, en cambio, tuvieron menos depresión severa al tener hijos $(\mathrm{p}<0,001$ y también $\mathrm{p}<0,001$ para menos depresión moderada), al tener más años de ejercicio profesional $(\mathrm{p}=0,038)$, al tener seguro social $(\mathrm{p}=0,003)$ y si un amigo se enfermó de COVID-19 $(\mathrm{p}=0,003)$ (Tabla 2).

Tabla 2. Análisis bivariado y multivariado de los factores socio-laborales asociados a padecer depresión moderada o severa entre los profesionales de la salud de la serranía peruana

\begin{tabular}{|c|c|c|}
\hline \multirow{2}{*}{ Variable } & \multicolumn{2}{|c|}{ Depresión moderada o mayor / RP (IC95\%) valor $p$} \\
\hline & Bivariado & Multivariado \\
\hline Sexo masculino & $0,91(0,56-1,46) 0,689$ & --- \\
\hline Edad (años)* & $1,00(0,94-1,05) 0,905$ & --- \\
\hline Tiene hijos & $0,50(0,48-0,52)<0,001$ & $0,49(0,42-0,57)<0,001$ \\
\hline Años que ejerce su profesión* & $0,99(0,94-1,05) 0,848$ & --- \\
\hline Horas que trabaja al día* & $0,90(0,84-0,97) 0,005$ & $0,92(0,84-1,01) 0,073$ \\
\hline Tiene seguro social & $0,48(0,24-0,95) 0,036$ & $0,59(0,32-1,10) 0,095$ \\
\hline Me enfermé yo de COVID-19 & $2,02(1,20-3,39) 0,008$ & $1,80(0,98-3,33) 0,060$ \\
\hline Un amigo se enfermó con COVID-19 & $0,56(0,20-1,55) 0,263$ & --- \\
\hline Algún familiar en casa se enfermó & $1,57(1,09-2,26) 0,016$ & $1,42(0,99-2,02) 0,055$ \\
\hline Familiar fuera de casa se enfermó & $0,82(0,43-1,57) 0,554$ & --- (2) \\
\hline \multirow[t]{2}{*}{ Algún familiar falleció por COVID-19 } & $1,31(0,78-2,20) 0,309$ & --- \\
\hline & \multicolumn{2}{|c|}{ Depresión severa o mayor / RP (IC95\%) valor p } \\
\hline Sexo masculino & $0,68(0,38-1,20) 0,186$ & --- \\
\hline Edad (años)* & $0,99(0,98-1,00) 0,012$ & $1,02(1,00-1,04) 0,017$ \\
\hline Tiene hijos & $0,47(0,31-0,70)<0,001$ & $0,50(0,34-0,74)<0,001$ \\
\hline Años que ejerce su profesión* & $0,98(0,96-0,99)<0,001$ & $0,98(0,97-0,99) 0,038$ \\
\hline Horas que trabaja al día* & $0,97(0,88-1,06) 0,497$ & --- \\
\hline Tiene seguro social & $0,27(0,17-0,44)<0,001$ & $0,45(0,27-0,77) 0,003$ \\
\hline Me enfermé yo de COVID-19 & $1,92(0,85-4,36) 0,118$ & --- \\
\hline Un amigo se enfermó con COVID-19 & $0,23(0,11-0,50)<0,001$ & $0,27(0,12-0,64) 0,003$ \\
\hline Algún familiar en casa se enfermó & $1,88(1,54-2,30)<0,001$ & $1,96(1,54-2,51)<0,001$ \\
\hline Familiar fuera de casa se enfermó & $0,34(0,12-0,97) 0,045$ & $0,51(0,13-1,98) 0,329$ \\
\hline Algún familiar falleció por COVID-19 & $0,53(0,12-2,40) 0,414$ & -- \\
\hline
\end{tabular}

Tuvieron menos ansiedad los que tuvieron hijos ( $\mathrm{p}=0,017$ para la moderada $\mathrm{y}<0,001$ para la severa), además, tuvieron menos ansiedad severa los que tuvieron a un familiar fuera de casa que se enfermó ( $\mathrm{p}=0,025)$. Por el contrario, tuvieron mayor ansiedad moderada los que trabajaban más horas al día ( $\mathrm{p}<0,001)$, severa si es que el encuestado se 
había enfermado $(\mathrm{p}=0,005)$ y de ambos tipos si es que algún familiar había fallecido ( $\mathrm{p}=0,002$ para la moderada y $<0,001$ para la severa) (Tabla 3 ).

Tabla 3. Análisis bivariado y multivariado de los factores socio-laborales asociados a padecer ansiedad moderada o severa entre los profesionales de la salud de la serranía peruana

\begin{tabular}{|c|c|c|}
\hline \multirow[t]{2}{*}{ Variable } & \multicolumn{2}{|c|}{ Ansiedad moderada o mayor / RP (IC95\%) valor } \\
\hline & Bivariado & Multivariado \\
\hline Sexo masculino & $1,04(0,88-1,22) 0,645$ & --- \\
\hline Edad (años)* & $0,98(0,95-1,02) 0,301$ & --- \\
\hline Tiene hijos & $0,66(0,48-0,92) 0,014$ & $0,64(0,44-0,92) 0,017$ \\
\hline Años que ejerce su profesión* & $0,98(0,95-1,01) 0,228$ & --- \\
\hline Horas que trabaja al día* & $1,08(1,06-1,11)<0,001$ & $1,08(1,06-1,11)<0,001$ \\
\hline Tiene seguro social & $0,84(0,49-1,46) 0,545$ & --- \\
\hline Me enfermé yo de COVID-19 & $1,04(0,73-1,46) 0,842$ & --- \\
\hline Un amigo se enfermó con COVID-19 & $0,83(0,52-1,34) 0,456$ & --- \\
\hline Algún familiar en casa se enfermó & $1,17(0,59-2,35) 0,654$ & --- \\
\hline Familiar fuera de casa se enfermó & $0,83(0,66-1,05) 0,113$ & --- \\
\hline \multirow[t]{2}{*}{ Algún familiar falleció por COVID-19 } & $1,32(1,06-1,65) 0,013$ & $1,47(1,15-1,88) 0,002$ \\
\hline & \multicolumn{2}{|c|}{ Ansiedad severa o mayor / RP (IC95\%) valor p } \\
\hline Sexo masculino & $0,97(0,64-1,48) 0,893$ & --- \\
\hline Edad (años)* & $0,97(0,95-0,98)<0,001$ & $0,98(0,96-1,01) 0,143$ \\
\hline Tiene hijos & $0,44(0,32-0,61)<0,001$ & $0,47(0,31-0,72)<0,001$ \\
\hline Años que ejerce su profesión* & $0,97(0,95-1,00) 0,092$ & --- \\
\hline Horas que trabaja al día* & $1,06(0,99-1,13) 0,098$ & --- \\
\hline Tiene seguro social & $1,46(0,97-2,19) 0,069$ & --- \\
\hline Me enfermé yo de COVID-19 & $1,49(1,17-1,90) 0,001$ & $1,63(1,16-2,30) 0,005$ \\
\hline Un amigo se enfermó con COVID-19 & $0,74(0,36-1,53) 0,413$ & --- \\
\hline Algún familiar en casa se enfermó & $0,94(0,86-1,04) 0,220$ & --- \\
\hline Familiar fuera de casa se enfermó & $0,61(0,49-0,76)<0,001$ & $0,67(0,47-0,95) 0,025$ \\
\hline Algún familiar falleció por COVID-19 & $1,87(1,20-2,92) 0,006$ & $2,12(1,40-3,19)<0,001$ \\
\hline
\end{tabular}

Tuvieron menos estrés los que habían ejercido la profesión más años ( $p<0,001$ para la moderada y 0,018 para la severa), pero tuvieron más estrés los que trabajaban más horas al día ( $\mathrm{p}<0,001$ para la moderada y 0,002 para la severa) y en su presentación moderada o mayor entre los que tenían un familiar fallecido (p<0,001) (Tabla 4).

Tabla 4. Análisis bivariado y multivariado de los factores socio-laborales asociados a padecer estrés moderado o severo entre los profesionales de la salud de la serranía peruana

\begin{tabular}{|c|c|c|}
\hline \multirow{2}{*}{ Variable } & \multicolumn{2}{|c|}{ Estrés moderado o mayor / RP (IC95\%) valor p } \\
\hline & Bivariado & Multivariado \\
\hline Sexo masculino & $0,57(0,29-1,09) 0,091$ & --- \\
\hline Edad (años)* & $0,98(0,96-0,99) 0,002$ & $0,99(0,97-1,01) 0,237$ \\
\hline Tiene hijos & $0,67(0,44-1,02) 0,060$ & -- \\
\hline Años que ejerce su profesión* & $0,97(0,96-0,98)<0,001$ & $0,99(0,98-0,99)<0,001$ \\
\hline Horas que trabaja al día* & $1,12(1,10-1,15)<0,001$ & $1,12(1,09-1,15)<0,001$ \\
\hline Tiene seguro social & No converge por poca data & --- \\
\hline Me enfermé yo de COVID-19 & $1,05(0,70-1,58) 0,819$ & --- \\
\hline Un amigo se enfermó con COVID-19 & $0,91(0,59-1,39) 0,651$ & --- \\
\hline Algún familiar en casa se enfermó & $1,08(0,63-1,87) 0,773$ & --- \\
\hline Familiar fuera de casa se enfermó & $0,71(0,41-1,20) 0,201$ & --- \\
\hline \multirow[t]{2}{*}{ Algún familiar falleció por COVID-19 } & $1,87(1,28-2,73) 0,001$ & $1,92(1,36-2,71)<0,001$ \\
\hline & \multicolumn{2}{|c|}{ Estrés severo o mayor / RP (IC95\%) valor p } \\
\hline Sexo masculino & $0,50(0,22-1,13) 0,098$ & --- \\
\hline Edad (años)* & $0,96(0,90-1,03) 0,220$ & --- \\
\hline Tiene hijos & $0,54(0,25-1,18) 0,125$ & --- \\
\hline Años que ejerce su profesión* & $0,96(0,92-0,99) 0,026$ & $0,96(0,93-0,99) 0,018$ \\
\hline Horas que trabaja al día* & $1,14(1,06-1,22) 0,001$ & $1,13(1,05-1,21) 0,002$ \\
\hline Tiene seguro social & No converge por poca data & --- \\
\hline Me enfermé yo de COVID-19 & $0,48(0,03-8,48) 0,617$ & --- \\
\hline Un amigo se enfermó con COVID-19 & $1,12(0,56-2,21) 0,742$ & --- \\
\hline Algún familiar en casa se enfermó & $1,40(0,20-9,63) 0,735$ & --- \\
\hline Familiar fuera de casa se enfermó & $0,71(0,22-2,34) 0,569$ & --- \\
\hline Algún familiar falleció por COVID-19 & $1,12(0,50-2,53) 0,780$ & --- \\
\hline
\end{tabular}




\section{Discusión}

Dentro de los hallazgos más relevantes se encontró que el tener hijos fue una variable que se asoció a una menor frecuencia de depresión moderada y severa, así como, a la ansiedad moderada y severa. Además, el tener más años ejerciendo la profesión de los encuestados se asoció a más frecuencia de depresión severa, estrés moderado y severo. También, se observó que tener seguro social y tener un amigo que se enfermó de COVID-19 redujo la aparición de casos de depresión severa. Por otro lado, el hecho de trabajar más horas al día en el centro de salud estuvo asociado a una mayor frecuencia de ansiedad moderada, estrés moderado y estrés severo. El tener mayor edad y algún familiar que vive en casa que se haya enfermado de COVID-19 se asociaron al tener depresión severa. Además, se encontró que si el participante tuvo algún familiar fallecido por COVID-19 tuvo más frecuencia de cuadros de ansiedad moderada, ansiedad severa y estrés moderado.

A nivel mundial, en el marco de la pandemia por COVID-19 se han identificado diversos problemas de la salud mental en el personal sanitario (PS) (Kang et al., 2020 a; Lai et al., 2020; Li et al., 2020 b). En China, un estudio realizado en 1257 trabajadores de salud de 34 hospitales de Hubei (la mayoría de Wuhan), asignados a salones con pacientes infectados por COVID-19, reportó que más del 70\% de enfermeras y médicos que fueron identificados con síntomas de angustia, el 50\% de trabajadores refirieron síntomas de depresión y se identificó ansiedad e insomnio en más de la tercera parte (Lai et al., 2020). Otro estudio, realizado en la misma provincia de Hubei, midió el impacto de la salud mental y las percepciones de la atención psicológica en el PS de un hospital de Wuhan, encontrándose que más del 30\% de trabajadores tuvieron alteraciones leves de depresión, ansiedad e insomnio; nuevamente la población más afectada fueron mujeres jóvenes (26-40 años) y en un 80\% laboraban fuera del área de alto riesgo (Kang et al., 2020 a). Finalmente un tercer estudio realizado en el mismo país, evaluó la repercusión traumática indirecta de 740 personas, donde más del 70\% eran enfermeras (234 de primera línea y 292 enfermeras no de primera línea) y el resto de la población fue el público en general, se observó que este último grupo y las enfermeras que no son de primera línea tuvieron grados más severos de trauma, en comparación con el otro grupo que si tuvo contacto con pacientes infectados, que laboran en el área de cuidados críticos y medicina interna (Li et al., 2020 b).

Con respecto a la depresión, el tener hijos estuvo asociado a una menor presentación de la depresión severa y moderada. Este hallazgo fue interesante, debido a que no se cuenta con estudios previos que hayan reportado esta variable en asociación al desarrollo de depresión. Por el contrario, se ha visto que en otros contextos la carga familiar o la responsabilidad parental son elementos que elevan los niveles de estrés de los padres y, en consecuencia, los predisponen a eventos depresivos (Arnal Remón, 2017). Por otro lado, se observó que los participantes con mayor edad y con familiares que enfermaron por COVID-19 tuvieron una mayor asociación de episodios de depresión severa. Esta posible relación puede tener su origen en el hecho de que los grupos de riesgos se encuentran entre las personas de más edad y con comorbilidades (Jain \& Yuan, 2020; Levin et al., 2020; Xu et al., 2020). En esa misma línea, la enfermedad de un familiar del círculo más cercano tiene la capacidad de poder afectar el ánimo de los individuos encuestados. Con respecto a esto último, Esquivel et al. desarrollaron un estudio en 197 familiares de pacientes hospitalizados y encontró que el 35\% desarrolló cuadros de depresión y que el $49 \%$ tuvo episodios ansiosos (Esquivel Molina et al., 2007).

En este estudio, los estados de ansiedad tanto moderados como severos fueron mucho más frecuente en aquellos participantes que tenían el antecedente de tener un familiar que había fallecido a causa del COVID-19. De manera similar, Pascual Fernández encontró, en un estudio realizado en enfermeras de la unidad de cuidados intensivos (UCI) del Hospital General Universitario Gregorio Marañón, que ante el ambiente de muerte y sufrimiento de sus pacientes se desarrollaron cuadros de depresión en un 19\% de las encuestadas, sobre todo, si el evento fatal ocurría en pacientes con menor edad (Pascual Fernández, 2011). Sin embargo, se carece de información fidedigna que evalué el impacto de la muerte de un familiar cercano en el desarrollo de ansiedad moderada o severa en diferentes contextos. Además, se observó que el haber tenido un antecedente personal de COVID-19 fue un factor de riesgo para el desarrollo de cuadros de ansiedad severa. De manera que, Lozano-Vargas reportó que el 29\% del personal de salud enfrentado a la infección por COVID-19 tuvo cuadros de ansiedad en el Perú (Lozano-Vargas, 2020).

En cuanto al estrés, uno de los hallazgos más relevantes fue que a más horas de trabajo existía mayor asociación con cuadros de estrés moderado y severo. Gómez Esteban realizó un estudio que encontró, al igual que nuestros resultados, que a más carga laboral el personal de salud tenía mayor predisposición a episodios de estrés y, en algunos casos, el desarrollo de patologías más complejas -como el síndrome de Burnout- (Gómez Esteban, 2004). Por otro lado, se observó que a más años de ejercicio profesional había menor frecuencia de desarrollar estrés moderado y severo. Probablemente, esta relación inversa entre la experiencia laboral y el estrés se deba a un mejor manejo de situaciones complicadas que se obtienen conforme pasen los años -y sobre todo en lugares de alta presión-. Sin embargo, Rodriguez Carbajal y De Rivas Hermosilla encontraron que a más años de trabajo se sufre una especie desgaste profesional que, en la mayoría de veces, desencadena episodios de estrés y síndrome de Burnout (Rodríguez Carvajal \& De Rivas Hermosilla, 2011). Todo esto debería estudiarse en investigaciones prospectivas, con una mayor muestra, con más sedes 
y que capturen una mayor cantidad de posibles variables que expliquen estas patologías (tanto para el estrés como para las otras medidas).

Las principales limitaciones de este estudio recaen en el sesgo de selección, debido a que la selección de la muestra fue de forma no probabilística. En ese sentido, los datos obtenidos en este estudio representan a una importante población del personal de salud del Hospital Nacional Ramiro Prialé Prialé, de la ciudad de Huancayo (en Perú). Sin embargo, los hallazgos obtenidos aquí sirven como precedente sobre la situación de la salud mental del personal de salud en una de las ciudades más populosas del Perú y en uno de los hospitales de referencia nacional ubicado en la sierra centro. Por otro lado, los resultados de esta investigación pueden ser aplicables o interpretables a otras poblaciones sanitarias en situaciones similares. Por lo que, futuras investigaciones deberán conducir propuestas más complejas para abordar esta temática, que a su vez sirvan como análisis de base para la intervención psicológica y psiquiátrica que se deberá dar a corto, mediano y largo plazo.

En conclusión, las características del estado de salud mental del personal de salud del hospital Nacional Ramiro Prialé Prialé fueron que los que tenían mayor edad o algún familiar cercano con antecedente de COVID-19 estaban más predispuestos a desarrollar cuadros de depresión severa. Además, el haber tenido un familiar que sucumbió a la pandemia del COVID-19 estuvo más relacionado a los cuadros de ansiedad moderada y severa. Finalmente, el tener mayor cantidad de horas de trabajo en un servicio de salud fue un factor de riesgo importante para el desarrollo de estrés moderado y severo.

\section{Conflicto de intereses}

Los autores declaran no tener conflicto de interés.

\section{Agradecimientos}

Agradecemos a todos los miembros del personal de salud del hospital en donde se realizó la investigación, que se dieron un poco de tiempo -a pesar de estar muy saturados- para poder ayudarnos con nuestra investigación.

\section{Referencias}

Arnal Remón, C. B. (2017). Estudio de las alteraciones emocionales y del bienestar psicológico de la pareja en diferentes etapas de la transición a la maternidad y la paternidad Tesis de Grado, Universidad Nacional de Educación a Distancia. Disponible en: http://e-spacio.uned.es/fez/view/tesisuned:Psicologia-Bcarnal (Acceso abril 2021)

Chang, C., Lo, S. C., Wang, Y.S. \& Hou, M.H. (2016). Recent insights into the development of therapeutics against coronavirus diseases by targeting $\mathrm{N}$ protein. Drug Discovery Today, 21(4): 562-572. https://doi.org/10.1016/j.drudis.2015.11.015

Chen, Q., Liang, M., Li, Y., Guo, J., Fei, D., Wang, L., He, L., Sheng, C., Cai, Y., Li, X., Wang, J., \& Zhang, Z. (2020). Mental health care for medical staff in China during the COVID-19 outbreak. The Lancet Psychiatry, 7(4): e15-e16. https://doi.org/10.1016/S2215-0366(20)30078-X

Daza, P., Novy, D. M., Stanley, M. A., \& Averill, P. (2002). The Depression Anxiety Stress Scale-21: Spanish Translation and Validation with a Hispanic Sample. Journal of Psychopathology and Behavioral Assessment, 24(3): 195-205. https://doi.org/10.1023/A:1016014818163

Epidemiology Working Group for NCIP Epidemic Response \& Chinese Center for Disease Control and Prevention. (2020). The epidemiological characteristics of an outbreak of 2019 novel coronavirus diseases (COVID-19) in China. Zhonghua Liu Xing Bing Xue Za Zhi, 41(2): 145-151. https://doi.org/10.3760/cma.j.issn.0254$\underline{6450.2020 .02 .003}$

Esquivel Molina, C. G., Buendía Cano, F., Villa Hernández, F., Ontiveros Martínez, R., Velasco Rodríguez, V. M., \& Martínez Mendoza, J. A. (2007). Ansiedad y depresión en familiares de pacientes hospitalizados. Medicina Interna de México, 23(6): 512-516.

Gómez Esteban, R. (2004). El estrés laboral del médico: Burnout y trabajo en equipo. Revista de la Asociación Española de Neuropsiquiatría, 90: 41-56.

Greenberg, N., Docherty, M., Gnanapragasam, S., \& Wessely, S. (2020). Managing mental health challenges faced by healthcare workers during covid-19 pandemic. BMJ, 368.m1211. https://doi.org/10.1136/bmj.m1211

Huang, J., Liu, F., Teng, Z., Chen, J., Zhao, J., Wang, X., \& Wu, R. (2020). Care for the Psychological Status of Frontline Medical Staff Fighting Against Coronavirus Disease 2019 (COVID-19). Clinical Infectious 
Diseases: An Official Publication of the Infectious Diseases Society of America, 71(12): 3268-3269. https://doi.org/10.1093/cid/ciaa385

Jain, V., \& Yuan, J.-M. (2020). Predictive symptoms and comorbidities for severe COVID-19 and intensive care unit admission: A systematic review and meta-analysis. International Journal of Public Health, 65(5): 533-546. https://doi.org/10.1007/s00038-020-01390-7

Kang, L., Li, Y., Hu, S., Chen, M., Yang, C., Yang, B. X., Wang, Y., Hu, J., Lai, J., Ma, X., Chen, J., Guan, L., Wang, G., Ma, H., \& Liu, Z. (2020 a). The mental health of medical workers in Wuhan, China dealing with the 2019 novel coronavirus. The Lancet. Psychiatry, 7(3): e14. https://doi.org/10.1016/S2215-0366(20)30047$\underline{X}$

Kang, L., Ma, S., Chen, M., Yang, J., Wang, Y., Li, R., Yao, L., Bai, H., Cai, Z., Xiang Yang, B., Hu, S., Zhang, K., Wang, G., Ma, C., \& Liu, Z. (2020 b). Impact on mental health and perceptions of psychological care among medical and nursing staff in Wuhan during the 2019 novel coronavirus disease outbreak: A crosssectional study. Brain, Behavior, and Immunity, 87:11-17. https://doi.org/10.1016/j.bbi.2020.03.028

Koh, D. (2020). Occupational risks for COVID-19 infection. Occupational Medicine, 70(1):3-5. https://doi.org/10.1093/occmed/kqaa036

Lagasse, J. (2020). Healthcare workers risk burnout, exposure in wake of coronavirus pandemic. Healthcare Finance News. Fisponoble en: https://www.healthcarefinancenews.com/news/healthcare-workers-risk-burnoutexposure-wake-coronavirus-pandemic (Acceso abril 2021)

Lai, J., Ma, S., Wang, Y., Cai, Z., Hu, J., Wei, N., Wu, J., Du, H., Chen, T., Li, R., Tan, H., Kang, L., Yao, L., Huang, M., Wang, H., Wang, G., Liu, Z., \& Hu, S. (2020). Factors Associated With Mental Health Outcomes Among Health Care Workers Exposed to Coronavirus Disease 2019. JAMA Network Open, 3(3):e203976. https://doi.org/10.1001/jamanetworkopen.2020.3976

Levin, A. T., Hanage, W. P., Owusu-Boaitey, N., Cochran, K. B., Walsh, S. P., \& Meyerowitz-Katz, G. (2020). Assessing the age specificity of infection fatality rates for COVID-19: Systematic review, meta-analysis, and public policy implications. European Journal of Epidemiology, 35(12):1123-1138. https://doi.org/10.1007/s10654-020-00698-1

Li, Q., Guan, X., Wu, P., Wang, X., Zhou, L., Tong, Y., Ren, R., Leung, K. S. M., Lau, E. H. Y., Wong, J. Y., Xing, X., Xiang, N., Wu, Y., Li, C., Chen, Q., Li, D., Liu, T., Zhao, J., Liu, M., ... Feng, Z. (2020 a). Early Transmission Dynamics in Wuhan, China, of Novel Coronavirus-Infected Pneumonia. The New England Journal of Medicine, 382(13):1199-1207. https://doi.org/10.1056/NEJMoa2001316

Li, Z., Ge, J., Yang, M., Feng, J., Qiao, M., Jiang, R., Bi, J., Zhan, G., Xu, X., Wang, L., Zhou, Q., Zhou, C., Pan, Y., Liu, S., Zhang, H., Yang, J., Zhu, B., Hu, Y., Hashimoto, K., ... Yang, C. (2020 b). Vicarious traumatization in the general public, members, and non-members of medical teams aiding in COVID-19 control. Brain, Behavior, and Immunity, 88:916-919. https://doi.org/10.1016/j.bbi.2020.03.007

Liang, Y., Chen, M., Zheng, X., \& Liu, J. (2020). Screening for Chinese medical staff mental health by SDS and SAS during the outbreak of COVID-19. Journal of Psychosomatic Research, 133:110102. https://doi.org/10.1016/j.jpsychores.2020.110102

Lozano-Vargas, A. (2020). Impacto de la epidemia del Coronavirus (COVID-19) en la salud mental del personal de salud y en la población general de China. Revista de Neuro-Psiquiatría, 83(1):51-56. https://doi.org/10.20453/rnp.v83i1.3687

Ministerio de Salud. (2020). Resolución Ministerial N 180-2020-MINSA que aprueba la Guía Técnica para el Cuidado de la Salud Mental del Personal de Salud en el contexto de COVID-19. MINSA. Disponible en: https://www.gob.pe/institucion/minsa/normas-legales/473129-180-2020-minsa (Acceso abril 2021)

Organización Mundial de la Salud. (2020). Nuevo coronavirus - China. WHO; World Health Organization. Disponible en: http://www.who.int/csr/don/12-january-2020-novel-coronavirus-china/es/ (Acceso abril 2021)

Organización Panamericana de la Salud. (2020). Consideraciones psicosociales y de salud mental durante el brote de COVID-19. OPS. Disponible en: https://www.paho.org/es/documentos/consideraciones-psicosocialessalud-mental-durante-brote-covid-19 (Acceso abril 2021)

Ornell, F., Halpern, S. C., Kessler, F. H. P., Narvaez, J. C. de M., Ornell, F., Halpern, S. C., Kessler, F. H. P., \& Narvaez, J. C. de M. (2020). The impact of the COVID-19 pandemic on the mental health of healthcare professionals. Cadernos de Saúde Pública, 36(4):e00063520. https://doi.org/10.1590/0102-311x00063520 
Pascual Fernández, M. C. (2011). Ansiedad del personal de enfermería ante la muerte en las unidades de críticos en relación con la edad de los pacientes. Enfermería Intensiva, 22(3):96-103. https://doi.org/10.1016/j.enfi.2011.01.004

Rodríguez Carvajal, R., \& De Rivas Hermosilla, S. (2011). Los procesos de estrés laboral y desgaste profesional (burnout): Diferenciación, actualización y líneas de intervención. Medicina y Seguridad del Trabajo, 57(1):72-88. https://doi.org/10.4321/S0465-546X2011000500006

Rolim Neto, M. L., Gomes Almeida, H., D’arc Esmeraldo, J., Bezerra Nobre, C., Pinheiro, W., Tavares de Oliveira, C. R., da Costa Sousa, I., Moreira Leite Lima, O. M., Nara Rolim Lima, N., Moreno Moreira, M., Tavares Lima, C. K., Gonçalves Júnior, J., \& Lima da Silva, C. G. (2020). When health professionals look death in the eye: The mental health of professionals who deal daily with the 2019 coronavirus outbreak. Psychiatry Research, 288:112972. https://doi.org/10.1016/j.psychres.2020.112972

Santarone, K., McKenney, M., \& Elkbuli, A. (2020). Preserving mental health and resilience in frontline healthcare workers during COVID-19. The American Journal of Emergency Medicine, 38(7):1530-1531. https://doi.org/10.1016/j.ajem.2020.04.030

Torales, J., O’Higgins, M., Castaldelli-Maia, J. M., \& Ventriglio, A. (2020). The outbreak of COVID-19 coronavirus and its impact on global mental health. The International Journal of Social Psychiatry, 66(4):317-320. https://doi.org/10.1177/0020764020915212

Xu, L., Mao, Y., \& Chen, G. (2020). Risk factors for 2019 novel coronavirus disease (COVID-19) patients progressing to critical illness: A systematic review and meta-analysis. Aging, 12(12):12410-12421. https://doi.org/10.18632/aging.103383

Zhu, N., Zhang, D., Wang, W., Li, X., Yang, B., Song, J., Zhao, X., Huang, B., Shi, W., Lu, R., Niu, P., Zhan, F., Ma, X., Wang, D., Xu, W., Wu, G., Gao, G. F., \& Tan, W. (2020). A Novel Coronavirus from Patients with Pneumonia in China, 2019. New England Journal of Medicine, 382(8):727-733. https://doi.org/10.1056/NEJMoa2001017 